\title{
Efeitos da Suplementação de Creatina na Força Máxima e na Amplitude do Eletromiograma de Mulheres
}

Fisicamente Ativas

\section{Effect of Creatine Supplementation in Maximal Strength and Electromyogram Amplitude of Physically Active Women}

\author{
Rômulo José Dantas Medeiros 2,3 \\ Alexsandra Araújo dos Santos ${ }^{2}$ \\ Alan de Carvalho Dias Ferreira ${ }^{2}$ \\ José Jamacy de Almeida Ferreira ${ }^{4}$ \\ Luis Carlos Carvalho ${ }^{4,5}$ \\ Maria do Socorro Cirilo de Sousa',2,3 \\ 1. Departamento de Educação Física \\ - Universidade Federal da Paraíba \\ (UFPB) - João Pessoa, PB. \\ 2. Grupo de Pesquisa em \\ Cineantropometria, Atividade \\ Física e Saúde, Desenvolvimento e \\ Desempenho Humano - GPCASD/CNPq. \\ 3. Mestrando do Programa Associado \\ de Pós-Graduação em Educação Física \\ UFPB/UPE - João Pessoa/Recife - PB/PE. \\ 4. Departamento de Fisioterapia \\ - Universidade Federal da Paraíba - \\ João Pessoa, PB. \\ 5. Centro Universitário de João Pessoa \\ - UNIPÊ - João Pessoa, PB.
}

\section{Endereço para correspondência:} Avenida Presidente Café Filho, 509, Bessa - 58035-180 - João Pessoa, PB. E-mail: romuloaquazul@yahoo.com.br

\begin{abstract}
RESUMO
A suplementação de creatina apresenta ação ergogênica na força muscular. Entretanto, não há consenso deste efeito na força isométrica máxima e na amplitude do eletromiograma (EMG). Assim, o objetivo deste estudo foi analisar os efeitos da suplementação de creatina na força isométrica máxima e na amplitude do EMG em mulheres fisicamente ativas. Vinte e sete mulheres (idade 23,04 \pm 1,82 anos, massa corporal 58,37 $\pm 6,10 \mathrm{~kg}$, estatura 1,63 $\pm 0,05 \mathrm{~m}$ e índice de massa corporal 21,93 $\pm 2,02 \mathrm{~kg} / \mathrm{m}^{2}$ ) foram designadas aleatoriamente para os grupos creatina $(\mathrm{GCr})(\mathrm{n}=13)$ e placebo $(\mathrm{GPL})(\mathrm{n}=14)$, os quais ingeriram diariamente, durante seis dias, $20 \mathrm{~g}$ de creatina mono-hidratada e $20 \mathrm{~g}$ de maltodextrina, respectivamente. Antes e depois da suplementação, a força foi medida em um dinamômetro isométrico durante contração isométrica voluntária máxima (CIVM) de extensão unilateral do joelho (três séries de 6 s intervaladas por 180s), com captação simultânea dos valores root mean square (RMS) do EMG obtido no músculo vasto lateral. A ANOVA de dois critérios de classificação (dois momentos $x$ dois grupos) e o teste de Wilcoxon foram utilizados na análise estatística dos dados paramétricos e não paramétricos ( $p<0,05)$. Após a suplementação, o GCr aumentou significativamente a força, com incrementos de 7,85\% ( $p=0,002), 7,31 \%(p=0,001)$ e 5,52\% ( $p=0,001)$ para a primeira, segunda e terceira séries, respectivamente. Para este mesmo grupo, os valores RMS aumentaram significativamente na terceira série $(p=0,026)$. O GPL não apresentou alterações significativas. Os resultados sugerem que a suplementação de creatina aumenta a força isométrica máxima e que a amplitude do EMG pode ser utilizada como indicador dessas alterações de desempenho.
\end{abstract}

Palavras-chave: creatina, eletromiografia, força muscular.

\begin{abstract}
Creatine supplementation has shown to enhance muscular strength. However, there is not a consensus on this effect on maximal isometric strength neither on electromyogram (EMG) amplitude. Thus, the aim of this study was to analyze the creatine supplementation effects on maximal isometric strength and EMG amplitude in physically active women. 27 women (age $23.04 \pm 1.82$ years, body mass $58.37 \pm 6.10 \mathrm{~kg}$, height $1.63 \pm 0.05 \mathrm{~m}$ and body mass index $\left.21.93 \pm 2.02 \mathrm{~kg} / \mathrm{m}^{2}\right)$ were randomly assigned in creatine $(\mathrm{CrG})(\mathrm{n}=13)$ or placebo group (PLG) ( $n=14)$. The CrG and PLG ingested $20 \mathrm{~g}$ /day of creatine and $20 \mathrm{~g} /$ day of maltodextrin during six days, respectively. The strength was measured before and after supplementation using a isometric dynamometer during a maximal isometric voluntary contraction (MIVC) of a unilateral knee extension (3 sets of $6 \mathrm{~s}$ with $180 \mathrm{~s}$ rest period), and the EMG was acquired from the vastus lateralis muscle and its amplitude quantified using the root mean square (RMS) values. Two-way ANOVA (2 groups $x 2$ moments) was used for parametric data and Wilcoxon test for non-parametric analysis $(p<0.05)$. After supplementation, $\mathrm{CrG}$ significantly enhanced strength, with increase of $7.85 \%(p=0.002) ; 7.31 \%(p=0.001)$ and $5.52 \%(p=0.000)$ for the first, second and third trials, respectively. In CrG, the RMS values significantly increased on the third trial ( $p$ $=0.026$ ). No changes were found in GPL. Results suggest that creatine supplementation enhance maximal isometric strength and EMG amplitude can be useful to identify these performance modifications.
\end{abstract}

Keywords: creatine, electromyography, muscle strength. 


\section{INTRODUÇÃO}

A utilização de suplementos nutricionais visando o aumento do desempenho físico é na atualidade uma estratégia bastante evidenciada no cotidiano de indivíduos fisicamente ativos, chegando a alcançar índices de 40\%,60\% e 100\% entre praticantes de atividade física não atletas, atletas de maneira geral e fisiculturistas, respectivamente. Porém, pesquisas não atestam o efeito ergogênico da maioria dos suplementos nutricionais comercializados, quando ingeridos conjuntamente a uma dieta saudável e adequada(1).

Dentre os suplementos, a creatina se apresenta em destaque por fazer parte de um seleto grupo de compostos nutricionais que tiveram sua ação ergogênica testada e comprovada no aumento dos níveis de força e potência muscular ${ }^{(2,3)}$. Contudo, as evidências não são acompanhadas de um consenso, podendo-se identificar resultados contraditórios associados a diferenças metodológicas entre modelos de pesquisa dos estudos (grupos independentes versus cross-over), tamanho da amostra, características dos sujeitos avaliados e às especificidades do protocolo de exercício utilizado (tipo de contração muscular, duração da atividade e intervalo entre séries) $)^{(4,5)}$.

Apesar de estudos não confirmarem a ação interveniente de alguns fatores, sugere-se que a característica do protocolo de avaliação interfere nos resultados obtidos, uma vez que maior efeito ergogênico da suplementação de creatina tem sido observado em contrações dinâmicas ${ }^{(6,7)}$, quando comparado às avaliações realizadas em contrações isométricas ${ }^{(8,9)}$. Possivelmente, estas diferenças de resultados estão relacionadas à redução da síntese de ATP pela via energética da creatina fosfato (ATP-CrP), apresentada pelo músculo esquelético em decorrência da elevada oclusão vascular provocada por esse último tipo de contração.

Como alternativa para a busca de resultados mais conclusivos referentes à ação da suplementação de creatina, a amplitude do eletromiograma (EMG), determinada pelos valores root mean square (RMS), tem sido considerada ferramenta útil, uma vez que estudos ${ }^{(10)}$ já demonstraram a sua capacidade de identificar alterações no padrão de recrutamento de unidades motoras, associadas às mudanças dos níveis de força. Dessa forma, testamos no presente estudo a hipótese do aumento em conjunto dos valores RMS e de força após a suplementação da creatina, tendo como objetivo a análise dos efeitos da suplementação de creatina sobre a força isométrica máxima de mulheres fisicamente ativas, fazendo uso do EMG como uma estratégia para a confirmação das mudanças de desempenho observadas.

\section{MÉTODOS}

a) Amostra e desenho experimental - 27 mulheres fisicamente ativas, estudantes do Curso de Educação Física da Universidade Federal da Paraíba (UFPB), com idades entre 20 e 27 anos (23,04 \pm 1,82 anos), foram designadas aleatoriamente para os grupos creatina $(\mathrm{Cr})(\mathrm{n}=13)$ e placebo (GPL) ( $n=14)$. Como critérios de inclusão amostral, foram consideradas elegíveis para o estudo as voluntárias que apresentaram as seguintes características: idade entre 18 e 30 anos, experiência mínima de seis meses em exercício com pesos, ser eumenorreica, não fumar, não ingerir bebidas alcoólicas, ter o membro inferior direito como dominante para as atividades de vida diária, não apresentar histórico de lesão musculotendínea ou articular no joelho e não estar fazendo uso de esteroides anabolizantes, assim como quaisquer suplementos nutricionais. Quanto à exclusão, foram retiradas da amostra as voluntárias que deram início ao estudo, mas ingeriram indevidamente os suplementos propostos $(n=3)$ e realizaram sessões de exercício com pesos direcionadas à musculatura do quadríceps femoral nos dias que antecederam as avaliações de força $(n=2)$.
Tabela 1. Características da amostra.

\begin{tabular}{|c|c|c|c|c|}
\hline \multirow[t]{2}{*}{ Variáveis } & \multicolumn{2}{|c|}{$\mathrm{GCr}(\mathrm{n}=13)$} & \multicolumn{2}{|c|}{ GPL $(n=14)$} \\
\hline & Média ( $\pm \mathrm{DP}) \mathrm{a}$ & Mín.b-Máx.c & Média ( $\pm \mathrm{DP}$ ) & Mín.-Máx. \\
\hline Idade (anos) & $23,54 \pm 1,94$ & $20-27$ & $22,57 \pm 1,65$ & $20-25$ \\
\hline \multicolumn{5}{|c|}{ Características antropométricas } \\
\hline $\operatorname{MCd}(\mathrm{kg})$ & $56,53 \pm 5,99$ & $48,10-70$ & $60,08 \pm 5,90$ & $53-71,63$ \\
\hline Estatura (m) & $1,62 \pm 0,5$ & $1,55-1,73$ & $1,64 \pm 0,05$ & $1,54-173,63$ \\
\hline IMCe $\left(\mathrm{kg} / \mathrm{m}^{2}\right)$ & $21,57 \pm 2,63$ & $17,89-28,04$ & $22,25 \pm 1,23$ & $20,70-23,93$ \\
\hline$\% G g(\%)$ & $23,54 \pm 4,82$ & $15,67-31,22$ & $25,97 \pm 3,71$ & $20,16-31,61$ \\
\hline MGh $(\mathrm{kg})$ & $13,48 \pm 3,60$ & $8,15-18,91$ & $15,71 \pm 3,39$ & $10,93-21,33$ \\
\hline MCMi (kg) & $43,18 \pm 3,79$ & $38,01-51,09$ & $44,43 \pm 3,52$ & $38,85-50,30$ \\
\hline \multicolumn{5}{|c|}{ Tempo de prática em exercício com pesos } \\
\hline Tempo (meses) & $13,61 \pm 3,54$ & $8-21$ & $15,21 \pm 3,40$ & $8-20$ \\
\hline
\end{tabular}

De acordo com a Resolução 196/96 do Conselho Nacional de Saúde do Brasil, o estudo foi aprovado pelo Comitê de Ética em Pesquisa do Centro de Ciências da Saúde da UFPB, sob o protocolo n 530/08, e as participantes assinaram um termo de consentimento livre e esclarecido, o qual continha informações sobre o tipo, objetivos e procedimentos a serem realizados no estudo.

Durante a pesquisa, as voluntárias, que compareceram ao laboratório em três ocasiões, em intervalos de sete dias cada, foram orientadas a não modificarem os seus programas habituais de exercício com pesos. Na primeira visita, as voluntárias realizaram uma familiarização ao teste de força e foram submetidas à avaliação antropométrica, além de terem recebido o recordatório habitual da dieta para ser preenchido e entregue na semana seguinte. Na segunda e terceira visitas, efetuadas antes e depois da suplementação, foi realizada a avaliação da contração voluntária isométrica máxima (CIVM) para a quantificação da força e a captação do EMG.

b) Avaliação antropométrica - A massa corporal foi mensurada utilizando uma balança digital (Soehnle ${ }^{\circledR}$ ) com resolução de $100 \mathrm{~g}$ e a estatura foi medida em um estadiômetro (Sanny ${ }^{\circledR}$ ) com precisão de um milímetro e espaço de uso de $0,40 \mathrm{~cm}$ a 2,20m. Após a quantificação dessas duas variáveis, calculou-se o IMC. Para a estimativa do percentual de gordura e a determinação dos subsequentes valores de massa de gordura absoluta, massa corporal magra e massa de gordura, a equação de Siri(11) foi empregada a partir do modelo de regressão de densidade corporal para três dobras cutâneas (adipômetro Sanny ${ }^{\circledR}$ ) proposto por Jackson e Pollock ${ }^{(12)}$

c) Avaliação nutricional - Sabe-se que o elevado consumo de carnes na dieta por vir a causar alterações na ação ergogênica da suplementação de creatina, devido a uma possível saturação desse composto no músculo esquelético(5). Dessa forma, com o objetivo de determinar possíveis diferenças no padrão de ingesta nutricional entre os grupos antes da suplementação, os hábitos alimentares da amostra foram avaliados por um nutricionista. Para tanto, aplicou-se o recordatório habitual da dieta, no qual as voluntárias ficaram responsáveis em anotar todos os alimentos consumidos e seus horários durante um período de três dias, sendo dois deles referentes a dias de semana e um ao final de semana. A partir do registro dietético e quantificação da distribuição dos macronutrientes foram calculados os valores médios de energia, em kcal, e os valores proporcionais de carboidratos, proteínas e lipídios em relação ao valor calórico total da dieta e à massa corporal. Para estes cálculos utilizou-se o software Nutrilife ${ }^{\circledR}$, versão 5G. 
d) Medida da força - A força isométrica foi avaliada pela determinação da CIVM do quadríceps femoral durante a extensão unilateral do joelho direito (dominante), em um dinamômetro isométrico desenvolvido pelo Núcleo de Estudos e Tecnologia em Engenharia Biomédica, Departamento de Engenharia Mecânica e o Laboratório de Análise do Movimento Humano do Departamento de Fisioterapia da UFPB, conectado a um transdutor de força e ao polígrafo digital BioMed ${ }^{\circledR(13)}$. Este protocolo foi composto por três contrações máximas de seis segundos, intervaladas por 180 segundos.

As voluntárias foram posicionadas sentadas e os ajustes de apoio para o tronco e membros superiores e inferiores foram feitos. $O$ joelho direito foi posicionado em um ângulo de $120^{\circ}$ e três faixas com velcro foram utilizadas para a fixação do corpo à cadeira, sendo empregadas para estabilização do quadril, membro inferior ipsilateral e tronco. Como forma de estímulo, comandos verbais foram padronizados em todos os momentos dos testes.

e) Captação do EMG - Após a tricotomia e assepsia da pele, eletrodos bipolares descartáveis de Ag/AgCl (Skintact), circulares, com $10 \mathrm{~mm}$ de diâmetro, foram fixados no ventre do músculo vasto lateral e afastados do ponto motor, de forma paralela à direção das fibras musculares, conforme preconiza o Surface EMG for a Non-invasive Assessment of Muscles (Seniam) ${ }^{(14)}$. Neste procedimento, utilizou-se como referência o ponto localizado a dois terços da distância entre a espinha ilíaca anterossuperior e a borda superior da patela. A distância entre os eletrodos foi estabelecida em $20 \mathrm{~mm}$ (centro a centro) e o eletrodo de referência foi fixado na tuberosidade da tíbia. Visando repetir com precisão os locais de colocação dos eletrodos na pele em todas as fases do estudo, os mesmos foram demarcados com pincéis de marcação permanente.

Para a captação do EMG foi utilizado um amplificador biológico com alta impedância de entrada, construído com base em um amplificador de instrumentação INA121 (Texas Instruments). A rejeição de modo comum do amplificador foi $>110 \mathrm{~dB}$, faixa de passagem de 10 a $470 \mathrm{~Hz}$ e ganho de 3.000. A amplitude do EMG foi determinada pela medida dos valores RMS, os quais foram obtidos nos dois segundos da CIVM em que foram observados os maiores valores de força em um patamar estável de contração. O software empregado na captação do sinal foi o polígrafo digital BioMed ${ }^{\circledR(13)}$.

f) Suplementação - Após a desginação aleatória das voluntárias para os grupos GCr e GPL, empregou-se o modelo duplo-cego para a administração dos suplementos. O GCr e o GPL ingeriram diariamente, durante seis dias, $20 \mathrm{~g}$ de creatina mono-hidratada ${ }^{(15,16)}$ e $20 \mathrm{~g}$ de maltodextrina, respectivamente, acondicionadas na forma de sachês e administradas em quatro partes iguais (5g) a cada quatro horas (8h00min, 12h00min, 14h00min e 18h00min). É pertinente destacar que $20 \mathrm{~g}$ de maltodextrina foram misturados à suplementação da creatina, para mascarar, com o seu alto poder adoçante, a presença desse suplemento nos sachês.

g) Análise estatística - Os dados foram analisados no pacote estatístico Statistical Package for Social Sciences (SPSS), versão 16.0 para Windows. Inicialmente, a normalidade e homocedasticidade das variáveis de interesse foram testadas pelas provas de Shapiro Wilk e Levene. Para as medidas de força, utilizou-se a análise de variância (ANOVA) de dois critérios de classificação (dois grupos $x$ dois momentos) na avaliação dos efeitos do experimento. Para os valores RMS, o teste não paramétrico de Wilcoxon foi empregado na identificação das possíveis mudanças de valores existentes entre as fases pré e pós-suplementação. No caso da avaliação nutricional realizada antes da suplementação, empregou-se o teste $U$ de Mann-Whitney na comparação das medidas entre grupos. O nível de significância adotado foi de $p<0,05$.

\section{RESULTADOS}

Os dados referentes à distribuição dos macronutrientes na dieta habitual do GCr e GPL indicam que, além de ambos possuírem níveis de ingestão recomendados para os macronutrientes ${ }^{(1)}$, não são identificadas diferenças significativas entre grupos para a ingestão diária por quilograma de peso de carboidratos $(p=0,610)$, proteínas ( $p=$ 207) e lipídios ( $p=0,752)$.

Após a utilização do suplemento, o GCr apresentou aumentos significativos na força (interação grupo $x$ momento para as três séries $\mathrm{e}$ média, respectivamente: $p=0,002, p=0,001, p=0,001$ e $p=0,001$ ), enquanto que o GPL não demonstrou alterações significativas em seus valores. Para as três séries e a sua média, o GCr aumentou em 7,85\% (de $77,13 \pm 12,91 \mathrm{kgf}$ para $83,19 \pm 15,82 \mathrm{kgf}$ ), 7,31\% (de 78,09 $\pm 13,17 \mathrm{kgf}$ para $83,80 \pm 14,61 \mathrm{kgf}$ ) $5,52 \%$ (de 78,26 $\pm 13,89 \mathrm{kgf}$ para $82,58 \pm 80,17 \mathrm{kgf}$ ) e $6,88 \%$ (de $77,83 \pm 13,14 \mathrm{kgf}$ para $83,19 \pm 14,74 \mathrm{kgf}$ ) (figura 1).

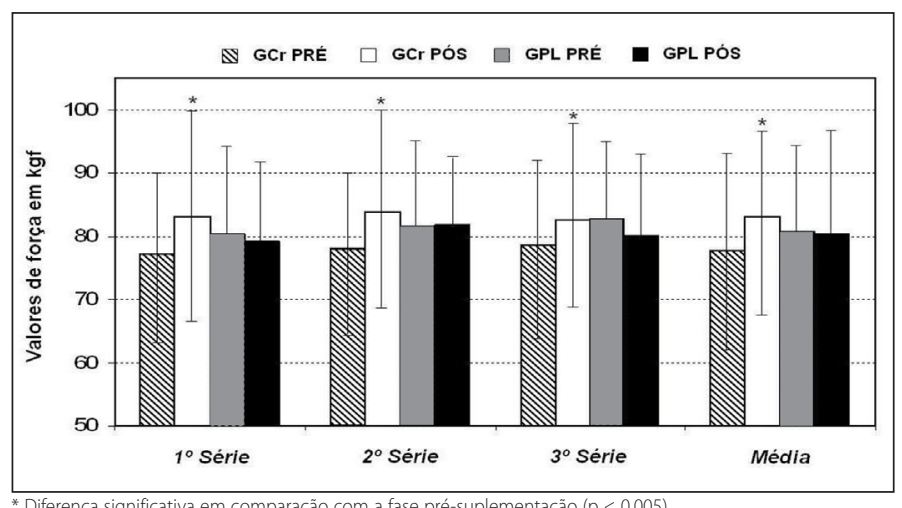

* Diferença significativa em comparaçăo com a fase pré-suplementação $(p<0,005)$.

Figura 1. Médias e desvios padrões dos valores da força em kgf antes e depois da suplementação.

Quanto aos valores RMS, no GCr foram observadas alterações significativas na terceira série $(p=0,026)$ e tendência de aumento na segunda série $(p=0,075)$ e na média ( $p=0,050)$ (figura 2$)$. O GPL não apresentou alterações em nenhuma de suas medidas.

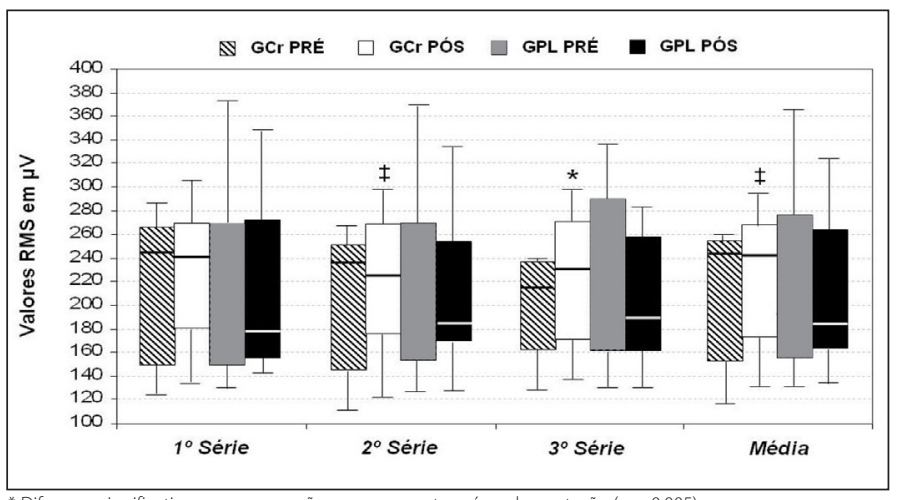

* Diferença significativa em comparação com momento pré-suplementação $(p<0,005)$. ₹ Tendência à diferença significativa em comparaçăo com a fase pré-suplementaçăo $(p<0,05)$.

Figura 2. Medianas e amplitudes interquartis dos valores RMS em $\mu \mathrm{V}$ antes e depois da suplementação.

\section{DISCUSSÃO}

No presente estudo foram observados aumentos significativos na CIVM e nos valores RMS do GCr, confirmando a hipótese de que há um aumento em conjunto destas medidas após a suplementação de creatina.

Na literatura são encontradas evidências indicando que a suplementação de creatina aumenta os níveis de força máxima, com predominância de resultados positivos para contrações dinâmicas/isotônicas 
e de pico de torque isocinético, e divergências relacionadas à força isométrica ${ }^{(4)}$. Em se tratando da CIVM, os resultados encontrados neste estudo corroboram os achados de Urbanski et al. ${ }^{(17)}$ que, analisando as alterações na CIVM de extensão de joelho em homens após a suplementação de creatina (20g/dia por cinco dias), verificaram aumentos significativos de 4,2\%.

Os aumentos constatados por Urbanski et al. ${ }^{(17)}$ foram menores que os encontrados neste trabalho (6,88\%). Tal resultado pode ser indicativo de que as mulheres apresentam maiores respostas à ação ergogênica da creatina quando comparadas aos homens, devido a possível menor saturação desse composto no músculo proveniente da alimentação. Contudo, os dados em destaque não permitem essa conclusão e comparação entre gêneros, uma vez que não foram avaliados em ambos os estudos os níveis de creatina por meio de biópsia.

Como resultados decorrentes do estudo, Urbanski et al. ${ }^{(17)}$ também constataram que mesmo sendo submetida a um protocolo de suplementação igual, a musculatura flexora dos dedos não apresentou aumentos de força, gerando a suposição de que grupos musculares localizados na região inferior do corpo podem ser mais suscetíveis ao aumento de desempenho. Estas diferenças entre grupos musculares podem estar relacionadas à maior solicitação dos membros inferiores nas atividades do cotidiano. Tal fato possibilitaria à musculatura do quadríceps, por exemplo, a obtenção de um maior nível de treinamento, o qual seria responsável pelo aprimoramento da capacidade do músculo em absorver a creatina e utilizá-la em contrações de alta intensidade após a suplementação(18).

Outro estudo, realizado por Kilduff et al..(19), também suporta os resultados do presente trabalho, apesar de ter analisado outro grupo muscular. Nesta pesquisa, após a separação dos indivíduos considerados responsivos ou não à suplementação de creatina, foram observados no grupo experimental (20g/dia de creatina por cinco dias) aumentos significativos da CIVM, enquanto que o placebo não alterou os seus valores. É importante observar que os autores também compararam a ingestão dos macronutrientes na dieta habitual antes da suplementação e não identificaram diferenças significativas entre grupos.

Por outro lado, diante das divergentes respostas provocadas pela suplementação de creatina na força isométrica máxima, é possível observar em homens idosos ${ }^{(20)}$ e adultos jovens destreinados ${ }^{(21)}$ valores de CIVM inalterados após a ingestão de $20 \mathrm{~g}$ de creatina por cinco dias. No caso dos adultos jovens, a diferença de resultados observada entre o presente trabalho e o estudo de Bemben et al.(21) pode ser explicada pelos distintos níveis de treinamento que caracterizaram as amostras. Os grupos avaliados por Bemben et al.(21), ao apresentarem baixos níveis de treinamento, podem ter adquirido aumentos de força provenientes do próprio protocolo de testes. Esse fato não foi observado no presente estudo, uma vez que aumentos de força ocorreram apenas no GCr.

As alterações nos níveis de força observadas em indivíduos fisicamente inativos, que se apresentam nas fases iniciais do exercício com pesos, ou qualquer outro de caráter resistido, são decorrentes de uma maior frequência de descarga de estímulos e sincronização das unidades motoras, na qual o tempo de liberação do potencial de ação e o recrutamento de unidades motoras são reduzidos; do aprimoramento da ação dos neurônios motores e sensoriais provenientes da medula espinhal; e do aperfeiçoamento da coordenação intermuscular, em que a ação negativa dos músculos antagonistas é inibida durante a realização de movimentos específicos ${ }^{(22)}$.

No caso dos estudos que avaliaram os efeitos da suplementação de creatina em contrações dinâmicas, como em testes de 1-RM e de pico de torque em dinamômetros isocinéticos, aumentos de força máxima são observados ${ }^{(23-25)}$. Ao se comparar a predominância de respostas er- gogênicas identificadas nos protocolos de contração dinâmica, colocase em questão os possíveis mecanismos fisiológicos que fundamentam as pesquisas a avaliarem a contração isométrica. Pesquisadores ${ }^{(17,21)}$ argumentam que nesse tipo de contração o grupo muscular avaliado é acometido por uma elevada oclusão vascular, que causa quedas de desempenho somadas a reduções nos níveis de ATP. Tal fato acarreta a redução da capacidade de ressíntese desse substrato através da CrP. Assim, ao serem identificados aumentos de desempenho, mesmo diante dessas respostas fisiológicas que dificultam o aporte e produção de energia, sugere-se que a via ATP-CrP aprimorou o seu funcionamento em decorrência do efeito ergogênico provocado pela suplementação de creatina.

No que diz respeito às alterações do EMG após o uso do suplemento de creatina, são escassos os estudos que utilizaram este sinal como variável para a identificação de mudanças de desempenho. Em se tratando da força máxima, a literatura reporta dados conflitantes, podendo encontrar depois da suplementação manutenção da força e dos valores $\operatorname{RMS}^{(20,25,26)}$ e aumento da força, com manutenção do RMS $^{(27)}$. Observa-se que nestes estudos não houve aumentos conjuntos dos valores RMS e de força, resultados estes que não estão de acordo com os dados obtidos no presente trabalho.

Os resultados de Van Leemputte et al. ${ }^{(25)}$ e Jakobi et al. ${ }^{(20,26)}$ confirmam a hipótese de que a manutenção da força máxima não é acompanhada pela alteração do EMG. No presente estudo, essa hipótese não foi confirmada, uma vez que o aumento da força foi acompanhado pela elevação dos valores RMS, estando esta mudança relacionada possivelmente ao maior recrutamento de unidades motoras e aumentos na frequência de disparo dos neurônios motores ${ }^{(10)}$.

Por sua vez, os resultados encontrados por Cramer et al. ${ }^{(27)}$ demonstraram que, mesmo sendo amplamente investigada, ainda não há consenso sobre a relação entre o EMG e a força(28). Inicialmente, a metodologia de avaliação proposta por Cramer et al. ${ }^{(27)}$ pode ter induzido essas contradições. Ao avaliar indivíduos fisicamente inativos em ambos os grupos, os aumentos de força máxima observados após o experimento tanto no grupo experimental (13\%) quanto no placebo (6\%), podem ter mascarado a identificação de mudanças significativas no EMG.

No presente estudo, os valores RMS foram sensíveis às alterações de força, mas não reproduziram igualmente os resultados, tendo em vista que a força aumentou significativamente em todas as séries e o valor RMS apenas em uma. Estes resultados sugerem que aumentos nos valores RMS são observados apenas nas situações em que um dos grupos não apresenta elevação da força, enquanto o outro demonstra aumentos significativos (neste caso, o GCr).

Muitos são os aspectos considerados na relação entre a força e o EMG. Referente aos fatores fisiológicos, a comparação de resultados entre estudos, quando analisadas a amplitude do EMG e a força, muitas vezes é inválida devido às diferentes características apresentadas pelos músculos avaliados. Neste sentido, a conformação estrutural das fibras musculares, bem como o seu comprimento e superficialidade em relação ao tecido adiposo, interferem diretamente na característica do EMG(28). Partindo do princípio que Cramer et al.(27) e o presente estudo avaliaram o mesmo músculo (vasto lateral) e seguiram uma padronização igual para a fixação dos eletrodos, pode-se inferir que a diferença de resultados é decorrente dos níveis de treinamento dos sujeitos das amostras.

Frente ao exposto, sugere-se que a suplementação de creatina aumenta a força isométrica máxima de mulheres fisicamente ativas e que a amplitude do EMG pode ser utilizada na identificação das alterações de desempenho observadas. Contudo, a aplicação desses resultados 
em contextos não laboratoriais deve ser feita de forma cautelosa, tendo em vista que estudos indicam possíveis diferenças na ação ergogênica da suplementação de creatina decorrentes do tipo de contração muscular e característica do músculo (tamanho, forma, entre outros), assim como dos níveis de treinamento dos indivíduos avaliados.

Como limitações metodológicas desta pesquisa, destaca-se a impossibilidade de se ter feito uso do modelo cross-over de estudo e de utilizar a suplementação de creatina por um maior período de tempo. O modelo cross-over permitiria ao final do experimento realizar comparações dos valores do EMG intra (pré e pós para um mesmo suplemento) e entre (comparação entre a suplementação com creatina e placebo) as fases de suplementação, já que todas as pessoas fariam uso tanto da creatina como do placebo em algum momento da pesquisa. Quanto à duração do experimento, a suplementação ingerida por um período maior (exemplo: 28 dias) possibilitaria a obtenção de dados referentes à ação ergogênica do suplemento para as fases de saturação e manutenção.

\section{CONCLUSÃO}

Pode-se concluir que a suplementação de creatina aumenta significativamente a força isométrica máxima de mulheres fisicamente ativas. A amplitude do EMG, por sua vez, mesmo não reproduzindo igualmente os aumentos de força observados em todas as séries de CIVM, apresentou acentuação significativa de valores em uma delas e tendência de aumento em outra, demonstrando ser uma variável capaz de indicar alterações de desempenho.

\section{AGRADECIMENTOS}

Os autores agradecem ao apoio financeiro do Programa de Apoio a Planos de Reestruturação e Expansão das Universidades Federais (REUNI).

Todos os autores declararam não haver qualquer potencial conflito de interesses referente a este artigo.

\section{REFERÊNCIAS}

6. Sociedade Brasileira de Medicina Do Esporte. Modificações dietéticas, reposição hídrica, suplementos alimentares e drogas: comprovação de ação ergogênica e potenciais riscos para a saúde. Rev Bras Med Esporte 2003;9:43-56.

7. Ziegenfuss TN, Rogers M, Lowery I, Mullins N, Mendel R; Antônio J, et al. Effect of a creatine loading on anaerobic performance and skeletal muscle volume in NCAA Division I athletes. Nutrition 2002;18:397-402.

8. Wright GA, Grandjean PW, Pascoe DD. The effects of creatine loading on thermoregulation and intermittent sprint exercise performance in a hot humid environment. J Strength Cond Res 2007;21:655-60.

9. Bemben MG, Lamont HS. Creatine supplementation and exercise performance. Sports Med 2005;35:107-25.

10. Gualano B, Benatti FB, Ferreira JCB, Franchini E, Brum PC, Lancha Junior AH. Efeitos da suplementação de creatina no exercício intermitente de alta intensidade: divergências e recomendações metodológicas. Rev Bras Cineantropom Desempenho Hum 2008;10:189-96.

11. Izquierdo M, Ibañez JJ, González-Badillo JJ, Gorostiaga EM. Effects of creatine supplementation on muscle power, endurance, and sprint performance. Med Sci Sports Exerc 2002;34:332-43.

12. Syrotuik DG, Bell GJ. Acute creatine monohydrate supplementation: a descriptive physiological profile of responders vs. non-responders. J Strength Cond Res 2004;18:610-7.

13. Bemben MG, Tuttle TD, Bemben DA, Knehans AW. Effects of creatine supplementation on isometric force-time curve character. Med Sci Sports Exerc 2001;33:1876-81.

14. Jakobi JM, Rice CL, Curtin SV, Marsh GD. Neuromuscular properties and fatigue in older men following acute creatine supplementation. Eur J Appl Physiol 2001;84:321-8.

15. De Luca CJ. The use of surface electromyography in biomechanics. J Appl Biomech 1997;13:135-63.

16. Siri WE. Body composition from fluid spaces and density: analysis for measuring body composition. In: Brozeck J, Henschel A, editors. Techniques for Measuring Body Composition. Washington: National Academy of Sciences, 1961;223-44.

17. Jackson AS, Pollock ML. Generalized equations for predicting body density of women. Med Sci Sports Exerc 1980;12:175-81

18. Carvalho LC, Lima RB, Duarte NB, Fernandes MR, Nóbrega AC, Batista, IVA. Software em Windows para Processamento, Armazenamento e Análise de Sinais Obtidos em Experimentos de Fisiologia. Congreso Latinamericano de Ingeniería Biomédica. Mazatlán, 1998;207-10. CD-ROM.

19. Hermes HJ, Freriks B, Disselhorst-Klug C; Rau G. Development of recommendations for SEMG sensors and sensor placement procedures. J Electromyogr Kinesiol 2000;10:361-74.
20. Cox G, Mujika L, Tumilty D, Burke L. Acute creatine supplementation and performance during a field test simulating match play in elite female soccer players. Int I Sport Nut Exerc Metab 2002;12:33-46.

21. Okudan N, Gokbel H. The effects of creatine supplementation on performance during the repeated bouts of supramaximal exercise. J Sports Med Phys Fitness 2005;45:507-11.

22. Urbanski RL, Loy SF, Vincent WJ, Vaspelkis III, BB. Creatine supplementation differentially affects maximal isometric strength and time to fatigue in large and small muscle groups. Int J Sport Nutr 1999;9:136-45

23. Izquierdo M, Ibañez JJ, González-Badillo JJ, Gorostiaga EM. Effects of creatine supplementation on muscle power, endurance, and sprint performance. Med Sci Sports Exerc 2002;34:332-43.

24. Kilduff JM, Vidakovic P, Cooney G, Twycross-Lewis R, Amuna P, Parker M, Paul L, Pitsiladis YP. Effects of creatine on isometric bench-press performance in resistance-trained humans. Med Sci Sports Exerc 2002;34:1176-83

25. Jakobi JM, Rice CL, Curtin SV, Marsh GD. Neuromuscular properties and fatigue in older men following acute creatine supplementation. Eur J Appl Physiol 2001;84:321-8

26. Bemben MG, Tuttle TD, Bemben DA, Knehans AW. Effects of creatine supplementation on isometric force-time curve character. Med Sci Sports Exerc 2001;33:1876-81.

27. Folland JP, Williams AG. The adaptations to strength training. Morphological and neurological contributions to increased strength. Sports Med 2007;37:145-68.

28. Rossouw F, Krüger PE, Rossouw J. The effect of creatine monohydrate loading on maximal intermittent exercise and Sport-specifics strength in well trained power-lifters. Nutrition Res 2000;20:505-14.

29. Syrotuik DG, Bell GJ. Acute creatine monohydrate supplementation: a descriptive physiological profile of responders vs. nonresponders. J Strength Cond Res 2004;18:610-7.

30. Van Leemputte M, Vandenberghe $K$, Hespel P. Shortening of muscle relaxation time after creatine loading. J Appl Physiol 1999;86:840-4.

31. Jakobi JM, Rice CL, Curtin SV, Marsh GD. Contractile properties, fatigue and recovery are not influenced by short-term creatine supplementation in human muscle. Exp Physiol 2000;85:451-60.

32. Cramer JT, Stout JR, Culbertson JY, Egan AD. Effects of creatine supplementation and three days of resistance training on muscke strength, power output and neuromuscular function. J Strength Cond Res 2007;21:668-77.

33. Staudenmann D, Roeleveld K, Stegeman DF, Van Dieen JH. Methodological aspects of SEMG recordings for force estimation - A tutorial and review. J Electromyogr Kinesiol 2009 [Epub ahead of print]. 